\title{
Vocalization and stapedius muscle activity evoked by local electrical stimulation of midbrain in the chicken (Gallus gallus)
}

\author{
Silvarosa Grassi ${ }^{1}$, Fabrizio Ottaviani ${ }^{2}$ and Domenico Bambagioni ${ }^{1}$ \\ 'Institute of Physiology and ${ }^{2}$ Institute of Otorhinolaryngology, University of Perugia, Perugia (Italy)
}

(Accepted 7 March 1989)

Key words: Stapedius muscle; Vocalization; Electrical stapedius muscle response; Different threshold; Field potential

\begin{abstract}
The organization of chicken mesencephalic areas from which stapedius muscle activity and vocalization can be differently elicited was studied. Our results show the existence of an area, around the mesencephalic 'calling area', from which stapedius muscle activity can be evoked independently of vocalization. Furthermore, low threshold 'vocalization loci' stimulation evokes field potentials in the stapedius-controlling area, due to the activation of stapedius-controlling neurons by vocalization neurons.
\end{abstract}

Unlike mammals, birds possess a single middle ear muscle (stapedius), located outside the middle ear cavity ${ }^{6}$, thus allowing the analysis of its activity without opening the middle ear cavity.

It is known that, unlike mammals, birds do not show an acoustic stapedial reflex ${ }^{3.9,11}$, while clear stapedius muscle contraction associated with vocalization is present in chickens ${ }^{1,2.3 .5}$.

Despite many hypotheses ${ }^{3.4}$, the physiological significance of stapedius muscle contraction in birds has not been fully clarified. In a previous paper $^{8}$, we reported that stapedius activity associated with evoked vocalization occurs in response to a central drive.

Furthermore, the finding that stimulation of discrete points within the chicken midbrain can elicit vocalization and stapedius muscle activity separately, suggested the existence of two midbrain neuronal populations projecting to the XIIth nucleus (controlling the syrinx) and to the VIIth nucleus (controlling the stapedius muscle).

The aim of the present work was to identify, by means of selective electrical stimulation, the loci within the chicken midbrain, from which vocalization and stapedius muscle activity could be differently elicited, and to verify whether an interaction between the mesencephalic calling and stapedial areas existed.

Twenty male leghorn chickens (Gallus gallus), 40-60 days old, of $1.8-2.0 \mathrm{~kg}$ body weight were used. Under urethane anesthesia ( $1 \mathrm{~g} / \mathrm{kg}$ b. wt., i.v.), a bipolar concentric stimulating electrode $(400 \mu \mathrm{m}$ in diameter) was stereotaxically implanted in a mesencephalic region between frontal planes A:2.50 and $A: 5 \cdot 00^{7}$. Each plane was tested at lateral and depth coordinates ranging from 0.50 to 6.00 by square pulses (0-100 $\mu \mathrm{A}, 0.5 \mathrm{~ms}$ duration) delivered at a rate of 100 pulses $/ \mathbf{s}^{10}$.

Vocalization was recorded using a microphone placed at a distance of about $7 \mathrm{~cm}$ from the chicken's beak (thus delaying the response by about $0.2 \mathrm{~ms}$ ), while electrical stapedius muscle response (EMG) was recorded by means of a bipolar electrode consisting of two $0.1 \mathrm{~mm}$ insulated (except at the tip) copper wires. EMGs (filtered between 0.1 and 10 $\mathrm{kHz})$ and vocalizations $(0.3-3.0 \mathrm{kHz})$ were recorded on a digital storage oscilloscope, transferred to a computer and stored on diskettes. Stimulating electrode tracks were localized according to appropriate histological processing of the brain.

Stimulation of the low-threshold 'vocalization loci' was then performed by single square pulses

Correspondence: S. Grassi, Instituto di Fisiologia Umana, Via del Giochetto, I-06100 Perugia, Italy. 
(0.08-0.1 ms duration). Field potentials were recorded from different loci within the mesencephalic stapedius-controlling area by means of $4 \mathrm{M}$ potassium acetate-filled pipettes (tip diameter 1-2 $\mu \mathrm{m}$, 10-30 $\mathrm{M} \Omega$ resistance). The reference electrode was inserted subcutaneously in the neck. The signals (filtered between 0.1 and $10 \mathrm{kHz}$ ) were recorded on a digital storage oscilloscope, transferred to a computer and subsequently processed using a program that calculated their average. The position of the stimulating and recording electrodes was subsequently inverted.

As previously reported ${ }^{8}$, electrical stimulation of the mesencephalic 'calling area' elicited vocalization which was invariably associated with a stapedius muscle EMG response. It was found that the threshold for both responses varied dependent on the points stimulated.

Upon stimulation of the central part of the mesencephalic 'calling area', the threshold for vocalization was lower than that for stapedius muscle EMG response (Figs. 1 and $2 \mathrm{~A}$ ), ranging from 9 to $70 \mu \mathrm{A}$. The latency (time from the first stimulus artifact to the beginning of the response) of the EMG was longer than that of the vocalization at low stimulus intensities (300 ms for EMG and $150 \mathrm{~ms}$ for vocalization) and decreased as the stimulus strength was increased, until it became shorter than that of the vocalization $(50 \mathrm{~ms}$ for EMG and $100 \mathrm{~ms}$ for vocalization) (Fig. 2A)

Following stimulation at more peripheral points within the 'calling area', the responses showed the same threshold, and the latency of EMG could be longer or shorter than that of the vocalization (Figs. 1 and 2B). When longer, it decreased with an increase of stimulus intensity, becoming shorter than that of the vocalization. In the other case, the first high muscle activity could be followed by a later one consequent on vocalization (Fig. 2B).

More peripherally, low stimulus intensities (26 $\mu \mathrm{A})$ elicited stapedius muscle EMG response alone, while vocalization appeared at higher stimulus levels (Figs. 1 and 2C). The EMG latency was always shorter than that of vocalization.

At the end, following stimulation at the most peripheral coordinates (probably outside the "calling area') stapedius muscle activity alone was elicited, showing a threshold ranging from 9.2 to $64 \mu \mathrm{A}$.
Vocalization was never obtained, regardless of the stimulus intensity (Figs. 1 and 2D).

These results strongly confirm our hypothesis that two different neuronal populations project from the midbrain to the XIIth and the VIIth nuclei respectively.

The area from which stapedius muscle activity alone could be elicited, was organized around the mesencephalic 'calling area' (Fig. 1). The wider representation of the 'calling area' was observed at A:3.50, while its extent was reduced moving both anteriorly and posteriorly. At A:3.00, a clear separation between medial and lateral vocalization systems appeared, and the stapedius-controlling area was represented peripherally to both systems. The organization of both areas was generally the same in all tested animals, though some individual differences concerning their borders could be detected, which could result in $0.5-1 \mathrm{~mm}$ shifts in mediolateral
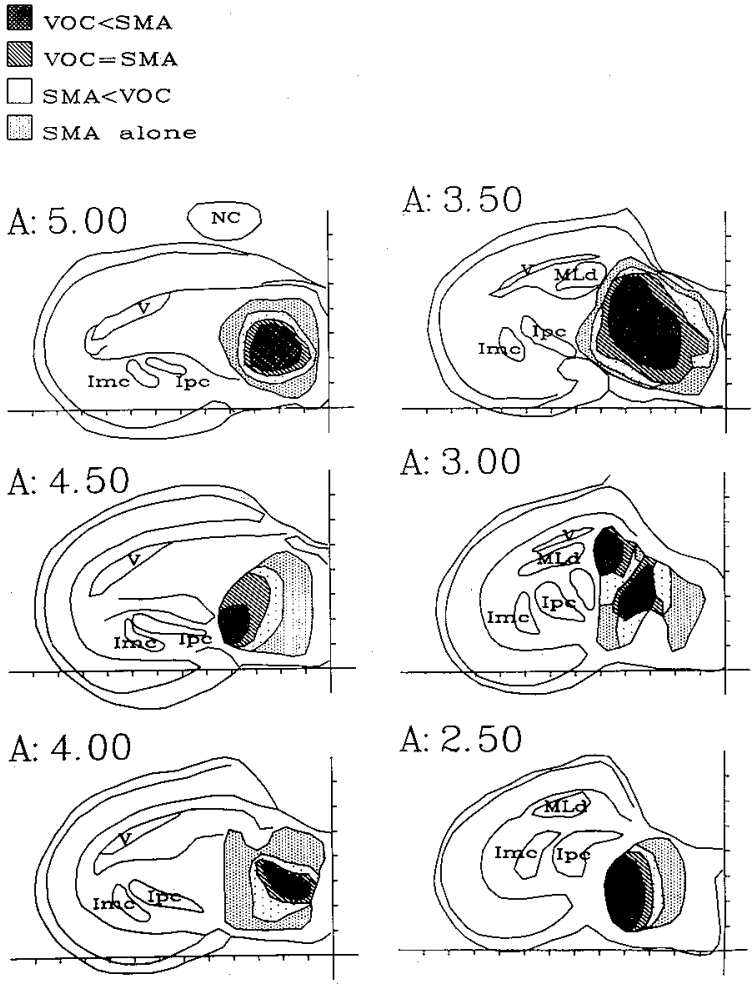

Fig. 1. Mesencephalic areas from which vocalization and stapedius muscle activity could be differently elicited are plotted on schematic sections of the chicken brain stereotaxic atlas?. On both axes, each division represents $1 \mathrm{~mm}$. $^{\mathrm{r}} \mathrm{-} \mathrm{c}$, Nucleus isthmi, pars magnocellularis; Ipc, Nucleus isthmi, pars parvocellularis; MLd, Nucleus mesencephalicus lateralis, pars ventralis; NC, Neostriatum caulalis; V, Ventriculus. 

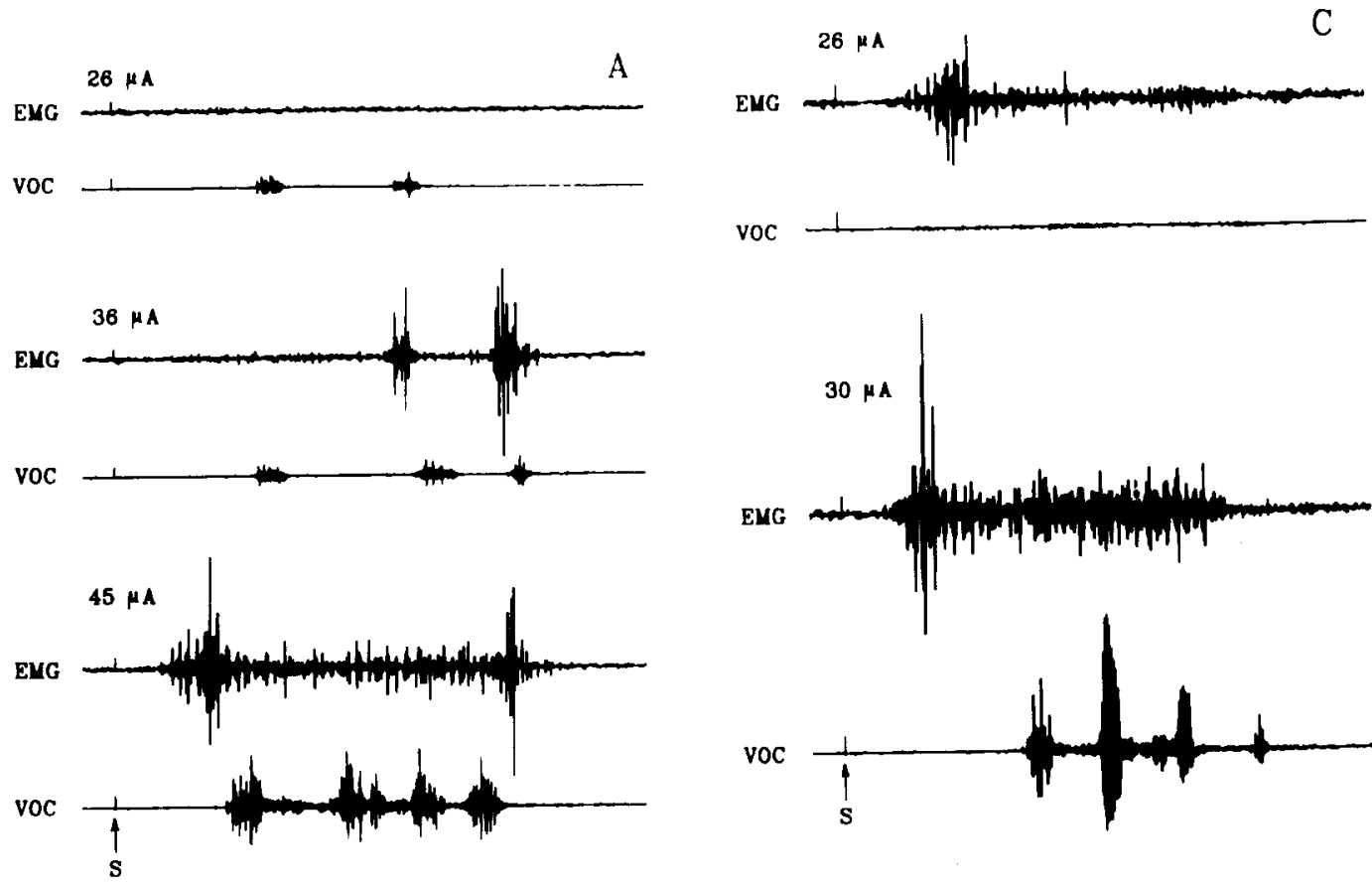

voc
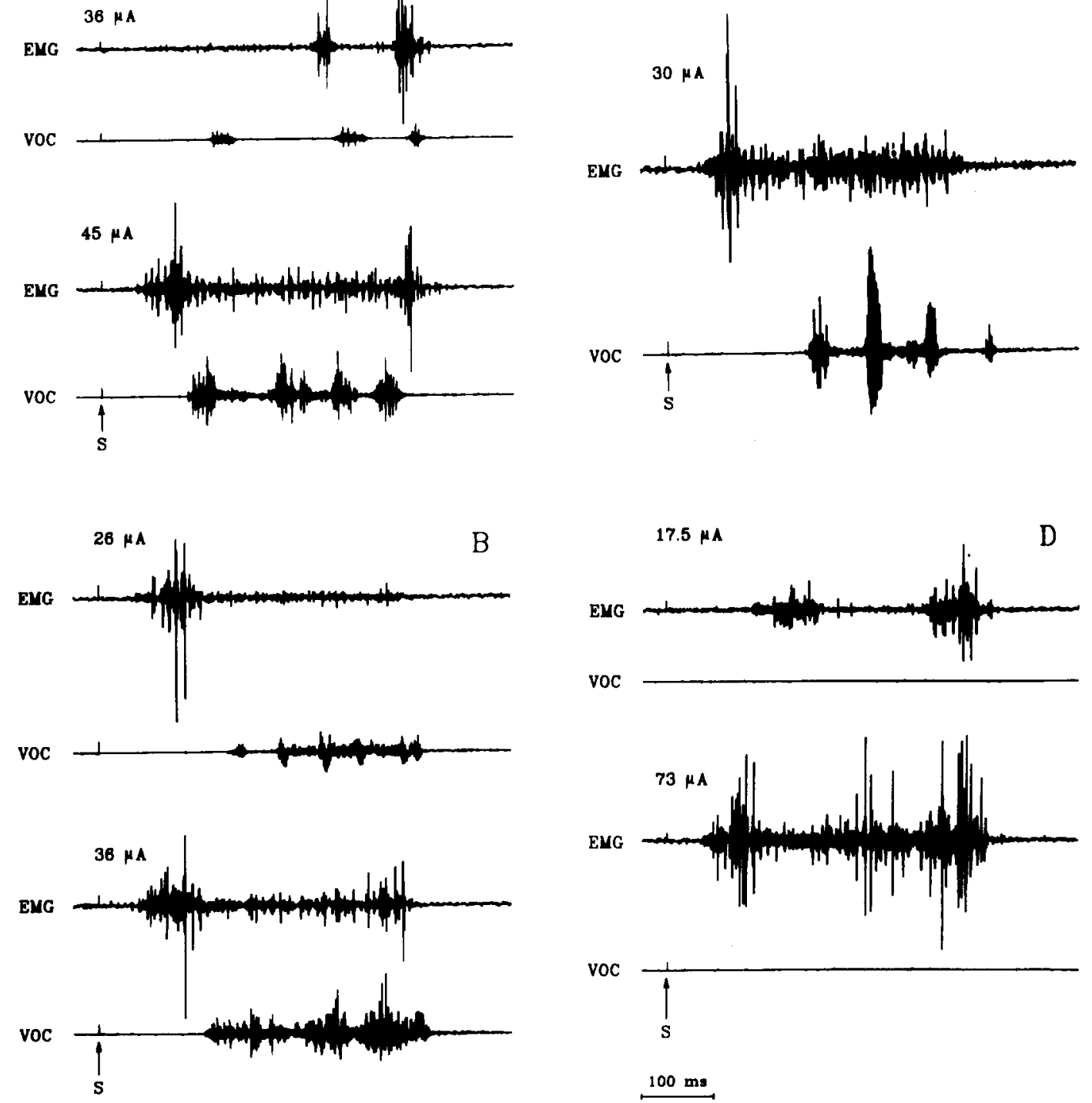

Fig. 2. Stapedius muscle EMG response (EMG) and vocalization (VOC) elicited by stimulating different mesencephalic loci where: A: the threshold for VOC is lower than that for EMG, B: both responses show the same threshold, C: the threshold for EMG is lower than that of VOC and D: stapedius muscle activity alone is elicited. Stimulus intensities $(\mu \mathrm{A})$ are indicated on the left of each record and the beginning of the stimulation by the $\mathrm{S}$. Time calibration applies to all records.

and/or dorsoventral directions.

The finding that stapedial activity showing latency and threshold higher than that for vocalization could be elicited by stimulating the 'calling area', suggests that stapedius-controlling neurons are driven by 'calling' ones at low stimulus intensities, but are directly activated when increasing stimulus intensities. In the latter condition, stapedius muscle contraction precedes vocalization, therefore a shorter conduction velocity of the stapedial effector system than that of the vocalization one can be predicted.

When moving the stimulating electrode toward 
$110 \mu \mathrm{A}$

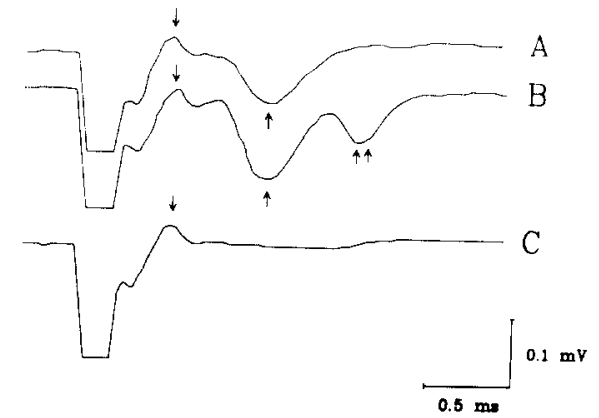

Fig. 3. A: field potential components (arrows) recorded from the mesencephalic stapedius-controlling area by stimulating the low-threshold 'vocalization loci'. B: field potential components recorded after high-frequency stimulation. $\mathrm{C}$ : antidromic response recorded from the 'vocalization-loci' by stimulating the stapedius-controlling area. Voltage and time calibrations apply to all records. Downward deflection indicates negativity.

the stapedius-controlling area, a direct activation can take place as well, because it is likely that both neuronal populations are coactivated. In this condition both responses showed the same threshold, and EMG latency could be longer or shorter than that of the vocalization at low stimulus intensities, according to a direct or secondary activation of stapediuscontrolling neurons. This also appears to be supported by the finding that at higher stimulus intensities an early (direct) and later (secondary) stapedius muscle EMG response can be simultaneously observed.

Furthermore, our results concerning the field potentials evoked in different loci of the stapediuscontrolling area by stimulating the low-threshold 'vocalization loci', provide physiological evidence for the existence of a projection from the 'calling' to the stapedius-controlling area.

The typical field potential consisted of two major components: a fast positive wave, with a latency of $0.25-0.3 \mathrm{~ms}$ and a negative one, characterized by a latency of $0.5-0.8 \mathrm{~ms}$ (Fig. 3A).

The nature of such components was investigated by the experiment illustrated in Fig. 3B, showing the effect of high-frequency stimulation (100/s, 2 s duration) of the 'vocalization loci' on the field potential elicited by a single shock $300 \mathrm{~ms}$ later. It can be seen that the amplitude of the second wave increased, whereas the early component was un- affected (Fig. 3B). Furthermore, a third later component (latency $1.2-1.5 \mathrm{~ms}$ ) was sometimes detectable (Fig. 3B). Displacement of the stimulating electrode outside 'vocalization loci' led to a disappearance of the responses, showing that the neural structures giving rise to the field potential are confined within the 'calling area'. The fact that the components of the field potentials are affected differently by high-frequency stimulation, suggests the presynaptic nature of the first wave and the postsynaptic nature of the second one. This hypothesis appears further suggested by the results obtained by inverting the position of the stimulating and recording electrodes. In this condition, by stimulating the mesencephalic stapedius-controlling area, only an antidromic response was recorded from 'vocalization loci' (Fig. 3C), whereas any later component was not detectable. The fact that the antidromic response was perfectly superimposable on the first component of the field potentials further supports its presynaptic nature, thus indicating a volley of propagated action potentials. Furthermore the finding that in this condition no later component was detectable, makes it unlikely that the second wave of the field potential represents the activation of a slower conduction pathway, so supporting its postsynaptic nature. Moreover, the latency of the second wave $(0.5-0.8 \mathrm{~ms})$ shows that it may represent the monosynaptic activation of the stapediuscontrolling neurons by the vocalization ones. The inconstant presence of a third component of the field potential can be explained as the result of a further neuronal recruitment, due to the tetanic stimulation of 'vocalization loci'. Our results provide evidence that stapedius-controlling neurons are unable to drive the vocalization ones, and that the vocalization elicited by stimulating the mesencephalic stapediuscontrolling area, is due to a current spread from stapedial to 'vocalization loci'.

Taken together our results bring support to the concept that vocalization-related stapedius muscle activity in chickens is due to the activation of a distinct mesencephalic stapedius-controlling area by the vocalization system.

This work was supported in part by a grant from the Italian Ministry of Education. 
1 Borg, E., Counter, S.A. and Rydquist, B., Contraction properties and functional morphology of the avian stapedius muscle, Acta Otolaryngol., 88 (1979) 20-26.

2 Borg, E., Counter, S.A. and Lännergren, J., Analysis of the avian middle ear muscle contraction by strain gauge and volume and impedance change measures, Comp. Biochem. Physiol., 71A (1982) 619-621.

3 Counter, S.A. and Borg, E., Physiological activation of the stapedius muscle in Gallus gallus, Acta Otolaryngol., 88 (1979) 13-19.

4 Counter, S.A. and Borg. E., The avian stapedius muscle. Influence of auditory sensitivity and sound transmission, Acta Otolaryngol., 94 (1982) 267-274.

5 Counter, S.A., Borg, E. and Lännergren, J., Basic contraction properties of the avian stapedius muscle, Acta Physiol. Scand., 111 (1981) 105-108.

6 Evans, H.E., Nomina anatomica avium, Organa sensum, final list of terms, Int. Comm. Avian Anat. Nomenclature
(ICAAN), 1975

7 Grassi, S., Magni, F., Maurizi, M. and Ottaviani, F., A Stereotaxic Atlas of the Brain of the Domestic Chick (Gallus gallus), Maggioli, Rimini, 1984.

8 Grassi, S., Magni, F. and Ottaviani, F., Mechanisms controlling vocalization-evoked stapedius muscle activity in chickens (Gallus gallus), J. Comp. Physiol., 162 (1988) 525-532.

9 Oeckinghaus, H. and Schwartzkopff, J., Electrical and acoustical activation of the middle ear muscle in a songbird, J. Comp. Physiol., 150 (1983) 61-67.

10 Phillips, R.E., Youngren, O.M. and Peek, F.W., Repetitive vocalization evoked by local electrical stimulation of avian brains. I. Awake chickens (Gallus gallus), Anim. Behav., 20 (1972) 689-705.

11 Wada, Y., Beiträge zur vergleichenden Physiologie des Gehororgans, Pflügers Arch., 202 (1924) 46-49. 